\title{
Analysis of TB-DM Collaboration Program at the Hospital in Semarang City, Indonesia
}

\author{
Sulistiyani $^{1, *}$, Bagoes Widjanarko ${ }^{1}$, Praba Ginandjar $^{2}$, K. Heri Nugroho ${ }^{3}$, Tri Dewi Kristini ${ }^{4}$, Sugeng Rianto ${ }^{4}$, Novia \\ Handayani $^{5}$ \\ ${ }^{1}$ Doctoral Program of Public Health, Public Health Faculty, Diponegoro University, Semarang - Indonesia \\ ${ }^{2}$ Department of Epidemiology, Public Health Faculty, Diponegoro University, Semarang - Indonesia \\ ${ }^{3}$ Department of Internal Medicine, Faculty of Medicine, Diponegoro University, Semarang-Indonesia \\ ${ }^{4}$ Central Java Provincial Health office, Semarang-Indonesia \\ ${ }^{5}$ Department of Health Promotion, Public Health Faculty, Diponegoro University, Semarang-Indonesia
}

\begin{abstract}
Indonesia contributes to $45 \%$ of TB cases in the world. Currently, Diabetes mellitus (DM) case is increasing. DM may worsen TB. The risk of developing latent into active TB increases with the presence of DM. Treatment failure in TB patients with DM is more frequent than non-DM. The aim of this study was to analyze of TB DM collaboration program at the one hospital in Semarang City. This was a qualitative study with content analysis. Informants consisted of internists, nurses, patients. This study showed there was a gap between technical guidance from minister of health and implementation of DM screening from TB patient. Besides, human resources factors also contribute to that gap.
\end{abstract}

Keywords: TB-DM; Hospital; Collaboration; Program.

\section{Introduction}

TB case is currently increasing. Similarly, the number of patients with DM is increasing. Based on WHO report (2018), it was estimated that there were 10 million people developed TB. Indonesia had $8 \%$ of TB cases from all around the world. In 2017, TB-caused death was about 1.6 million. In Indonesia, there were 842,000 of TB suspects and 116,000 TB-caused death in 2017 [1]. TB problem gets heavier with DM. DM will weaken the immune system, increasing the risk of developing latent TB into active TB. From 2010 to 2016, TB patients were diabetic with an annual prevalence increased from $12.5 \%$ to $18.7 \%$ [2].

In Pulmonary Diseases Center, Semarang City, Prevalence of HBA1c $\geq 7$ was $29 \%$ [3]. Reported TBDM patients had higher mortality (10.3\%) than non DM patient $(7.6 \%)$ [2]. Diabetes also a risk factor for adverse tuberculosis treatment outcomes, including death [4]. Since 2015 there has been the Technical Guidance of TB-DM case finding at the Secondary Referral Health Facility (FKRTL) [5]. However, in health facilities have not been implemented, until the socialization of TB-DM collaboration by the City Health Office of Semarang in May 2017.

The aim of this research was to analyze TB-DM collaboration program at hospital in Semarang City, center of Java Indonesia.

\section{Method}

This research was a qualitative method with the secondary data observation step in one selected hospital, indepth interviews to the informants Informants in this study were internist-DM, internist-TB, nurse in DOTs clinic, nurse in TB-DM program, analyst, TB-DM patients. Additional informants in this study were health assurance (BPJS). Data were collected through 3 techniques, they are:

1. Observation, to analyze initial condition related to TB- DM program in chosen health facilities

2. In-depth interview, to identify the obstacles of integrated TB-DM program and factors contributed to the problems

3. Focus group discussion, to identify an alternative model of integrated TB-DM program development

This study also conducts dissemination of the research result in order to make a commitment to developing the integrated TB-DM program. Data were analyzed using content analysis based on the result of in-depth interview and focus group discussion..

Ethical Clearance was approved by Ethics Commission Public Health Faculty, Diponegoro University. No. 221/EC/FKM/2017.

* Corresponding author: sulistiyani@lecturer.undip.ac.id 


\section{Result and Discuss}

Secondary data was from medical records in hospital. showed an increase in the number of TB cases and an increase in the number of DM cases. But the cases of TB-DM did not increase. This showed that there was a "gap" in TB-DM.

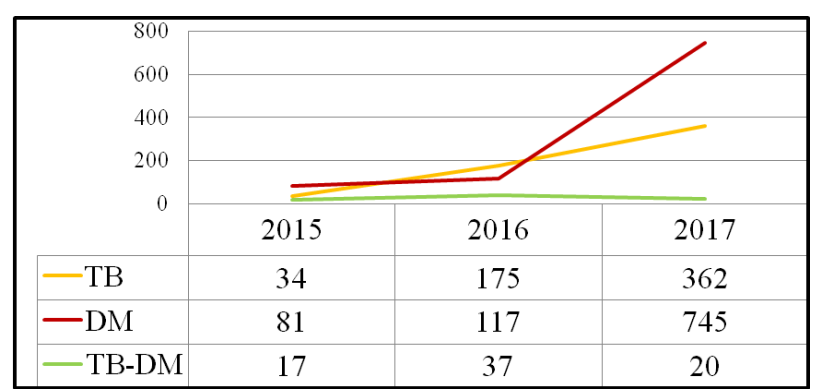

Fig. 1. The number of cases TB, DM, TB-DM in Hospitals.

By 2015 and 2017 the proportion of TB-DM found among TB cases is higher than the proportion of TB-DM found among DM cases. But otherwise in 2016, the proportion of TB-DM among DM cases was higher than the proportion of TB-DM among TB cases.

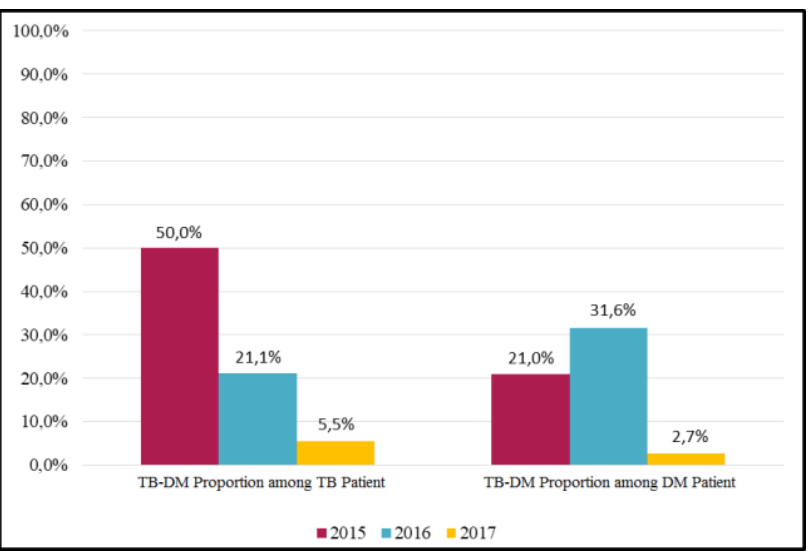

Fig. 2. The proportion of TB-DM among TB patients and among DM patients.

The other research said that DM was strongly associated with TB in an urban setting in Indonesia. This may have implications for TB control and patient care [6].

In-depth interview result that DM screening is done by checking blood sugar, both plasma glucose and fasting plasma glucose. Besides that, by observing the symptoms that present from the patient and ask a history of previous DM and family history of DM. Here is a statement from the informant.

"We also ask the patient before the doctor came here (TB-DOTS clinic), while we check the vital sign, we do anamnesis to the patients by asking if there is any history of DM in their family, also if patients have any symptoms related to DM." (Informant 4)

This research result showed that three of the study sites already performed DM screening to all TB patients, especially to new TB patients. This result is supported by a study conducted in India which showed that the screening of DM among patients with TB is encouraged because it could improve case detection, early treatment, and prevention of DM complications [7].

Since July 2017, DM screening was performed for all TB patients without exception, especially in new TB patients in Indonesia. Indonesia has a guideline for tuberculosis control, DM screening among TB patient is included in tuberculosis control [5].

Factors that contribute to the problems of collaboration TB-DM program. Such as in practice, screening for DM in $\mathrm{TB}$ patients does not always do well. Informant statement explains there were some TB patients who refuse to be screened for DM, due to fear and ignorance in patients. However, after doctors explain more about the benefits of screening for DM, patients understand and want to be screened.

“...the patient doesn't want to be screened. But we always explain the importance of glucose test, sputum test and radiology test. It's important for the patient. Generally, after we give explanation, patient understand and want to be screened" (informant 5)

TB screening performed to all DM patients by observing TB symptoms. The symptoms and indications of TB on DM patients are coughs, especially cough with phlegm for more than 2 weeks, intermittent fever, night sweats without activities, weight loss, out of breath or pain when breathing or feeling heavy on one side of the chest, enlarged lymph nodes (extrapulmonary TB). After TB symptoms are found on DM patients, then a chest X-ray is examined to find lung abnormalities. However, on the TB monitoring and evaluation meeting in August, there was new information that TB screening on DM patients will be done by TCM-Tes Cepat Molekuler (Rapid Molecular Test).

TB screening on DM patients in hospital only performed by medical staff who attended the socialization of integrated TB-DM program in May 2017. One informant stated that the information has been delivered to other medical staff, but it was unknown if it was already implemented or not. Following is the statement:

"...maybe some medical staff have not got the information by other staff...this has to be socialized to other medical staff, not only me who knows about this. (Informant 7)

There was a gap in the implementation of integrated TBDM program in this hospital. DM patients' visits are numerous, so nurses and doctors sometimes do not have time to fill out the TB screening form in DM patients.

The point is...maybe from the patients' number... why the patients just a little in this hospital...maybe it is because not all doctors know about this.... Well it is, individual, I said it has not spread yet, um, socialized to all KSM members in the internist..." (Informant 7) 
The problem that emerges in this hospital was that the huge number of DM patients caused the nurses and doctors do not have enough time to fill the form of TB screening to DM patients. Prevalence of diabetes is increasing globally, but most rapidly in low-income and middle-income countries where tuberculosis is a grave public health problem [4].

There was no internal legal from hospital that strengthens the implementation of TB-DM collaboration program, for example, SOP. So the implementation of collaboration TB-DM program cannot be done well by all health workers in hospital.

"...if the rule from Ministry of Health like Permenkes already exists, we expect an intern rule or policy from the Director of the hospital also exist, so we would really want to perform it. Conducting a national program..." (Informant 7)

\section{Conclusion and sugestion}

Conclusion from this research were:

There was a gap in the implementation of collaboration TB-DM program in hospital. There were problems of implementation of collaboration TB-DM program in health facility. Factors that contribute to the problems of collaboration TB DM program in health facilities are : human resources and internal regulation

\section{Suggestion :}

Important to make internal regulation management of TB-DM such as Standard Operational Procedures of TB$\mathrm{DM}$ in the hospital. Important to dissemination of TBDM report for the health workers in hospital. Important to disseminationof the Technical Guidance of TB-DM case finding at the Secondary Referral Health Facility for the organization of internist (lungs and endocrinologist).

This research was supported by the grant of Indonesia Ministry of Health, Directorate General of Disease Prevention and Control with research contract no: HK.0301/1/1599/2017.

\section{References}

1. WHO. Global Tuberculosis Report 2018. (2018).

2. D.T. Nguyen and E.A. Gravis, Diabetic trends and associated mortality in tuberculosis patients in Texas, a large population-based analysis, Tuberculosis 116, S59-S65 (2019).

3. P. Ginanjar, L.D. Saraswati, and B. Widjanarko, Profile of glycated-hemoglobin, antioxidant, vitamin and cytokine levels in pulmonary tuberculosis patients: A cross sectional study at Pulmonary Diseases Center Semarang City, Indonesia, Biomedical Journal 39, 354-360 (2016).

4. K. Lonnroth, G. Roglig, and AD. Haries, Improving tuberculosis prevention and care through addressing the global diabetes epidemic: from evidence to policy and practice, Lancet Diabetes Endocrinol Sep;2(9), 730-739 (2014)

5. Ministry of Health. National Guidelines for tuberculosis control. (Jakarta, 2014).

6. B. Alisjahbana, et al., Diabetes mellitus is strongly associated with tuberculosis in Indonesia. Int $\mathrm{J}$ Tuber Lung Dis 10, 696-700 (2006)

7. N. Boyilla, and S. Madas, Relationship between diabetes mellitus and pulmonary tuberculosis and the outcome of treatment, Int J Adv Med. Aug;3(3), 504-508 (2016). 